\title{
CLINIQUIZ
}

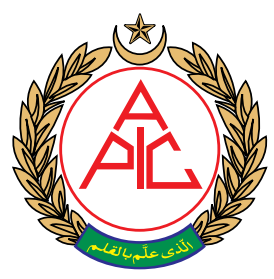

\section{COVID-19: General considerations and anesthetic management}

\author{
Pranav Bansal ${ }^{1}$, Mayuri Gupta ${ }^{2}$, Ishrat Yousuf ${ }^{2}$ \\ ${ }^{1}$ Professor \& Head*, ${ }^{2}$ Resident, Department of Anesthesiology \& Critical Care, \\ BPS Govt Medical College, Khanpur Kalan, Sonipat, Haryana (India) \\ DOI: https://doi.org/10.35975/apic.v24i1.1236
}

Corona virus emerged as a surprise to the world, just three months back, and within the matter of only a few weeks engulfed whole of the globe in a state of despair and fear. From Wuhan to New York, the clinicians found them on the forefront of a world war like condition. Most of the healthcare systems found them ill prepared to deal with the evergrowing number of victims. As expected, the brunt of this catastrophe had to bear by the intensivists, anesthesiologists, pulmonologists and the nursing staff, besides almost every trade and discipline of the workers in a hospital. (Please select one option in the questions given below).

\section{Q 1: The corona virus is a type of:}
(A) Non-enveloped negative sense RNA virus
(B) Enveloped positive sense RNA virus
(C) Enveloped positive sense DNA virus
(D) Non-enveloped negative sense DNA virus

Q 2: Which of the following is not a mode of transmission of COVID-19 infection?
(A) Fecal-oral
(B) Droplets
(C) Vector-borne
(D) Fomites

Q 3: The gold standard investigation for diagnosis of COVID-19 infection in a symptomatic patient is:
(A) RT PCR
(B) CT scan
(C) Coombs test
(D) CXR

Q 4: Which of the following is correct regarding N95 mask?

(A) The mask is made up of 6 protective layers.
(B) It has the capacity to filter $95 \%$ of particles of size greater than 0.3 microns in aerosols.

(C) The N95 mask is oil resistant.

(D) CDC recommends use of N95 mask for general public to prevent Corona virus infection.

Q 5: Which of the following is incorrect regarding general preventive measures to be taken by an anesthesiologist for Covid-19 infection?

(A) Hand wash should be performed frequently with soap and water.

(B) Mock drills for correct donning and doffing should be performed.

(C) Intubation may be performed using normal surgical mask by any health care provider.

(D) The recommended concentration of isopropyl alcohol in hand sanitizer is $70 \%$.

Q 6: For initial resuscitation of adults with COVID-19 and shock, fluid therapy for resuscitation includes which of the following?
(A) Buffered \& balanced crystalloids
(B) Unbuffered \& unbalanced crystalloids
(C) Colloids
(D) Blood

Q 7: Which of the following is true regarding conduction of surgery in suspected/confirmed case of COVID-19?

(A) The positive pressure system and air conditioners must be turned on for maintaining ambient OT temperature and environment

(B) At least 1 heat and moisture exchange (HME) filter must be connected between tracheal tube and breathing circuit and the other between expiratory limb and anesthesia machine 
(C) General anesthesia is to be preferred wherever possible due to compromised pulmonary status of such patients

(D) Use of only direct laryngoscope is indicated for intubation

Q 8: Which of the following is incorrect regarding the guidelines for disinfection with reference to COVID-19 infection?

(A) Wiping of surfaces of furniture, equipment and floor must be done with $2-3 \%$ of hydrogen peroxide spray.

(B) All the suspected cases should be planned in a single OT in continuation to minimize the risk of exposure.

(C) Sodium hypochlorite may also be used to decontaminate OT.

(D) Disposable videolaryngoscope blades should be re-sheathed by operator using gloved hands and discarded after use.

$Q$ 9: The recommendations in mechanically ventilated patients with COVID-19 with ARDS are:

(A) Low tidal volume with plateau pressures < $30 \mathrm{cmH}_{2} \mathrm{O}$

(B) High tidal volume with plateau pressures $>30$ $\mathrm{cmH}_{2} \mathrm{O}$

(C) Low tidal volume with plateau pressures $<30 \mathrm{cmH}_{2} \mathrm{O}$

(D) High tidal volume with plateau pressures $>30$ $\mathrm{cmH}_{2} \mathrm{O}$

Q10:Cytokinestorm syndromeisahyperinflammatory state characterized by all except:

(A) Multi-organ failure

(B) Secondary hemophagocytic lymphohistiocytosis

(C) Elevated cytokines

(D) Thrombocytopenia

\section{ANSWERS}

A 1. (B)

A 2(C): Human to human transmission occurs by droplet, contact, fomites and feco-oral route. The average incubation period of Covid-19 virus in humans is 114 days.

A 3(A): COVID-19 diagnosis is based on RT-PCR testing of nasopharyngeal and oropharyngeal swabs along lower respiratory tract samples whenever possible. Real-time reverse transcriptase polymerase chain reaction (RT-PCR) is the gold standard for similar viral infections, including SARS.
A 4(D): The respirator letter rating is N: Not oil resistant, R: Resistant to Oil and P: Oil Proof. The mask is made up of 4 layers of polypropylene and cellulose/polyester. CDC does not recommend its use for general public to prevent Corona virus infection.

A 5(C): Intubation should be performed by the most experienced person within shortest possible time only after wearing N95 mask. The endotracheal tube cuff should be inflated prior to ventilation there by, avoid delivery of aerosol to the health care providers involved in the task of performing intubation.

A 6(A): For the acute resuscitation of adults with COVID-19 and shock, guidelines recommend use of crystalloids over colloids (strong recommendation, moderate quality evidence) and buffered/balanced crystalloids are preferred over unbalanced crystalloids (weak recommendation, moderate quality evidence).

A 7(B): Since majority of operation theatres in India are not negatively pressurized, the positive pressure system and air conditioning must be turned off. Prefer regional anesthesia, where ever possible. Videolaryngoscope (if available) can be used to improve intubation success. Also, it may increase the distance between the patient's airway and that of the anesthesiologist who performs the intubation.

A 8(D): A minimum of one hour interval is planned between cases to allow OT staff to send the patient back to the ward, conduct thorough decontamination of all surfaces, screens, keyboard, cables, monitors and anesthesia machine with 2 to $3 \%$ hydrogen peroxide spray disinfection, $25 \mathrm{~g} / 1$ chlorine disinfectant, or $75 \%$ alcohol wiping of solid surfaces of the equipment and floor. The hydrogen peroxide vaporizer is an added precaution to decontaminate the OT.

Resheath the laryngoscope blade immediately post intubation with the outer glove worn by the operator.

A 9(A): No specific guidelines for management of patient of COVID-19 with ARDS on mechanical ventilation have been issued till the writing of this questionnaire. General guidelines recommend using low tidal volume ventilation (Vt $4-8 \mathrm{~mL} / \mathrm{kg}$ of predicted body weight), over higher tidal volumes $(\mathrm{Vt}>8 \mathrm{~mL} / \mathrm{kg}$ ), targeting plateau pressures (Pplat) of $<30 \mathrm{~cm} \mathrm{H} 2 \mathrm{O}$ (strong recommendation, moderate quality evidence), higher PEEP strategy, over a lower PEEP strategy (weak recommendation, low quality evidence).

A 10(D): Cytokine Storm Syndrome is a hyperinflammatory state that is characterized by fulminant multi-organ failure and elevation of cytokine levels. A recent study from China showed that COVID-19 is associated with a cytokine elevation profile that is reminiscent of secondary hemophagocytic lymphohistiocytosis (HLH) 


\title{
REFERENCES
}

1. Malhotra N, Joshi M, Datta R, Bajwa

SJ, Mehdiratta L. Indian society of anaesthesiologists (ISA national) advisory and position statement regarding COVID-19. Indian J Anaesth
2020;64(4):259-63

2. Alhazzani $\mathrm{W}$, Møller $\mathrm{MH}$, Arabi YM, Loeb M, Gong MN, Fan E, et al. Surviving Sepsis Campaign: guidelines on the management of critically ill adults with Coronavirus Disease 2019 (COVID-19). Intensive Care Med (2020). Available online. [Free full text] DOl: https://doi. org/10.1007/s00134-020-06022-5

\section{MY MOST MEMORABLE PATIENT}

\section{Anticipated difficult intubation in a septic shock patient}

\author{
Dr S. Shakir Hasan \\ Manchester (UK)
}

Difficult intubation can be a real challenge for any anesthesiologist. It is particularly hazardous and much more difficult to manage in an emergency situation.

We present a case of a 72 years old male patient who was brought to ER with stridor and increasing shortness of breath. He was unstable hemodynamically on presentation, so was managed with adrenaline/salbutamol nebs, I/V fluids and intermittent boluses of vasopressors in the ER. ENT referral was also sought who tried a quick nasal scope, but couldn't come to any conclusion. During neck examination they could feel a mass in the upper part of the neck and made a provisional diagnosis of a pharyngeal abscess. Portable chest $\mathrm{x}$-ray was unremarkable and there was no time to do a CT neck to confirm the diagnosis due to the patient's worsening condition. It was decided to rush the patient to OR for an awake fibre-optic intubation in the presence of ENT team in case if there was a need to do an emergency tracheostomy. The OR was prepared accordingly and the patient's upper airway was sprayed and nebulized with lignocaine. A very small dose of remifentanil infusion was started for better tolerance of the procedure. Patient remained in an extremely critical condition with a systolic blood pressure $60-70 \mathrm{mmHg}$, despite of $\mathrm{I} / \mathrm{V}$ fluids and metaraminol infusion. He also required intermittent boluses of 25 mics of adrenaline. There was no time for central venous access.

The plan was modified under the circumstances and it was decided to use a glidescope first to assess the airway. Patient tolerated it very well. Fortunately he was intubated easily. Patient was then stabilized by getting the central venous access and received the noradrenaline and adrenaline infusions. He was then transferred to ICU. His CT neck was done later, which was inconclusive. Chest $\mathrm{x}$-ray in ICU did show bilateral infiltrates, probably it was the cause of his dyspnea and septic shock, but the cause of his stridor remained unclear. In due course, he died of multi-organ failure.

In this case a difficult airway was anticipated due to dyspnea plus stridor, but a rapidly worsening condition due to septic shock (bilateral pneumonia) led us to prepare for even the ultimate management - the surgical airway, as the margin of error was very slim.

DOI: https://doi.org/10.35975/apic.v24i1.1239 